\title{
Editorial
}

\section{Applications of Medical Physics}

\author{
Salvatore Gallo ${ }^{1,2}$ (D) and Ivan Veronese ${ }^{1,2, * \mathbb{D}}$ \\ 1 Dipartimento di Fisica "Aldo Pontremoli", Università degli Stud di Milano, Via Celoria 16, \\ 20133 Milano, Italy; salvatore.gallo@unimi.it \\ 2 Istituto Nazionale di Fisica Nucleare (INFN), Sezione di Milano, Via Celoria 16, 20133 Milano, Italy \\ * Correspondence: ivan.veronese@unimi.it
}

check for

updates

Citation: Gallo, S.; Veronese, I. Applications of Medical Physics. Appl. Sci. 2022, 12, 1852. https:// doi.org/10.3390/app12041852

Received: 18 January 2022

Accepted: 5 February 2022

Published: 11 February 2022

Publisher's Note: MDPI stays neutral with regard to jurisdictional claims in published maps and institutional affiliations.

Copyright: (C) 2022 by the authors. Licensee MDPI, Basel, Switzerland. This article is an open access article distributed under the terms and conditions of the Creative Commons Attribution (CC BY) license (https:// creativecommons.org/licenses/by/ $4.0 /)$.

\section{Introduction}

Since the discovery of X-rays, the use of the principles and methods of physics in medicine has contributed to the improvement of human health. Physics-based techniques have been progressively developed and optimized with the aim to support physicians to formulate prompt diagnoses and to set up the effective treatments of several diseases.

Together with the continuous advances in the clinical procedures making use of ionizing radiations and related instruments, several other emerging and powerful resources are becoming more and more important. Tools such as ultrasounds for oncological and neurological therapeutic practices, nanoparticles, nanotechnologies for theragnostic applications, and artificial intelligence for quantitative medical imaging are just a few examples of the rich panorama of instruments currently available to the Medical Physicists.

Today, medical physics is a mainstream discipline recognized worldwide, with applications covering numerous areas of medicine and whose impact and scientific results deserve to be disseminated among the community of the applied sciences.

With this mission in mind, the scope of this Special Issue of Applied Sciences, entitled "Applications of Medical Physics", is to collect original research manuscripts describing cutting-edge physics developments in medicine and their translational applications, as well as reviews providing update on the latest progresses in this field.

A total of 28 manuscripts have been submitted to the Special Issue, and 20 of them have been accepted for publication. The final collection includes 17 original research manuscripts and 3 reviews by authors from 9 different countries. The published papers cover several areas, spanning from radiation therapy, nuclear medicine, radiology, dosimetry, radiation protection, and radiobiology.

A quick overview and general classification of the manuscripts is given below.

\section{Contributions}

Various contributions of this Special Issue deal with the research toward physicsbased innovative approaches for the treatment of oncological diseases or, more in general, propose the optimization of the methods currently employed in several clinical practices. External radiation therapy, brachytherapy, and theragnostic approaches are considered, the improvement of patient outcomes being the final and common aim of the studies. The attempt to reveal and understand the biological or radio-biological mechanisms governing such approaches is also often highlighted.

In this context, Crapanzano et al. propose in their review [1] an overview of the inorganic and hybrid nanoparticles of interest for X-ray-based oncological treatments. The authors point out how the physicochemical properties of the nanoparticles, together with the choice of surface functionalization and targeting strategies, affect the key parameters of external X-ray radiation therapy and X-ray activated photodynamic therapy in terms of energy deposition and total delivered dose in target tissues.

The optimization of radiotherapy procedures in the cohort of patients affected by esophageal cancer is considered in the manuscript by Tai et al. [2]. In fact, this study aims to 
evaluate the effect of radiation to the Epicardial Adipose Tissue (EAT) on survival outcomes in patients with esophageal cancer receiving neoadjuvant chemoradiotherapy followed by esophagectomy. The authors highlight the importance to consider EAT as an organ at risk (OAR) and propose the index of "EAT radiation exposure intensity" as a biomarker of survival outcomes in these patients.

Brachytherapy is the main field considered by Jo et al. [3]. In their manuscript, the authors highlight how potential applicator displacements can lead to significant changes in dose distribution during cervical brachytherapy treatments. To face this problem, a robust optimization scheme developed using a genetic algorithm combined with a median absolute deviation as a robustness evaluation function is proposed.

In the frame of eye proton therapy optimization for personalized treatments, Petringa et al. [4] compare Relative Biological Effectiveness (RBE) values obtained from in vitro experimental data with predictions made by the Local Effect Model, Monte Carlo approaches, and semi-empirical models based on Linear Energy Transfer (LET) experimental measurements. Their results evidence how a Treatment Planning System based on an RBE and LET prediction could improve the estimation of the radiobiological response of the treated neoplasia as well as the surrounding healthy tissues.

Finally, in the field of nuclear medicine, Urbano et al. [5] investigate the possible role of $\left[{ }^{99 \mathrm{~m}} \mathrm{Tc}\right] \mathrm{Tc}-S e s t a m i b i$ in the regulation of biological processes involved in breast cancer progression such as proliferation and apoptosis. Indeed, ex vivo and in vitro data about the correlation between sestamibi uptake and apoptosis suggest the possible role of sestamibi in the regulation of the pathophysiological processes involved in breast cancer. In conclusions, the authors suggest that the evidence of the accumulation of sestamibi in breast cancer cells and the subsequent mitochondrial damage can open new clinical perspectives on the use of this radiopharmaceutical in both the diagnosis and treatment of breast cancers.

Another subject that unites various contributions of this Special Issue is the research toward the optimization of the procedures employed in radiological imaging and the characterization of the related instruments and tools with the primary aim of patient dose reduction.

In this context, Albano et al. [6] compare radiation doses and imaging performances of an EOS imaging (Paris, France) system with those of conventional radiography and computed tomography (CT) in the case of the detection and measurement of enchondromas. The analysis suggests that low-dose EOS digital radiographic evaluation has the same capability as conventional radiography to detect and measure enchondroma-like inserts on a phantom. Thanks to its lower radiation dose, this imaging method could be particularly useful in the follow-up of patients affected by multiple enchondromas.

The study of Muhammad et al. [7] focuses on the possibility of reducing organ doses in pediatric CT chest-abdominal pelvis examination by optimizing the CT protocols. The effect of scan parameters such as tube voltage, tube current, slice collimation, pitch, and tube current modulation on image quality indices and organ doses are accordingly investigated. The results suggest that a significant potential reduction in the dose for such type of diagnostic examinations is achievable.

Dose optimization in CT scans is also considered in the manuscript by Barca et al. [8]. The authors highlight the need for an exhaustive characterization of noise properties in CT imaging with iterative reconstruction (IR) algorithms for assessing the actual dosereduction potential of this technique, as well as its possible limitations for specific clinical applications. In their in-phantom study, the authors carry out a voxel-based assessment of noise properties in CT imaging with adaptive statistical iterative reconstruction (ASiR) and adaptive statistical iterative reconstruction-V (ASiR-V) for different blending levels of reconstruction and contrast objects. Their results confirm the potential of ASiR-V and ASiR in reducing noise as compared with conventional filtered back projection approaches, suggesting, however, that the use of pure ASiR-V or ASiR might be suboptimal for specific clinical applications. In conclusion, authors recommend a voxel-based characterization of noise 
properties when dealing with a novel IR algorithm or when comparing the performances of different IR algorithms.

Quantitative imaging is another recurrent topic in various contributions of this Special Issue. Actually, the quantitative analysis of medical images proved to be a powerful approach for improving diagnostic and treatment strategies, as well as for developing predictive models.

Texture analysis is performed by Savini et al. [9] to characterize the impact of the acquisition angle on Digital Breast Tomosynthesis (DBT) images. The authors conclude that the DBT acquisition angle affects the textures extracted from DBT images, and this dependence should be considered when establishing baselines for classifiers of malignant tissue. Therefore, texture analysis in DBT has the potential to be an alternative to other quantitative techniques such as model observer methods for scoring and comparing DBT images.

Quantitative analyses are also increasingly used in nuclear medicine, both for diagnostic and therapeutic purposes. Di Martino et al. [10] highlight how the Partial Volume Effect (PVE) is the most important factor of loss of quantification in Nuclear Medicine. Therefore, in order to achieve an accurate quantification of the radioactivity concentration, PVE has to be considered and compensated, especially in small structures. In this context, the authors present a new approach for the correction of PVE, using a post-reconstruction process starting from a mathematical expression, which only requires the knowledge of the Full Width at Half Maximum of the Point Spread Function of the imaging system.

In the domain of low-field MR-guided radiotherapy for cervical cancer, Cusumano et al. [11] evaluate the performance of the radiobiological parameter Early Regression Index $\mathrm{ERI}_{\mathrm{TCP}}$ in predicting pathological complete response. The results suggest that ERI $\mathrm{TCP}_{\text {, }}$ calculated by combining the tumor volume measured on the MR images acquired at simulation and during therapy, is a promising response biomarker for cervical cancer. If validated on a larger cohort of patients, the use of this index can represent a valuable tool to personalize the treatment strategy in the context of the cervical cancer, moving towards the anatomical and functional preservation of the irradiated tissues.

Mazzilli et al. [12] propose a semi-automatic segmentation method to characterize the lungs of COVID-19 patients with respiratory syndrome. The approach is based on a robust, operator-independent identification of threshold Hounsfield Unit (HU) values that distinguish three regions of the lungs with intuitively clear functional meaning. The next step toward a fully automatic segmentation is also considered by combining the implemented method with an atlas of CT images of COVID-19 patients. The quantitative analysis carried out on the HU histograms paves the way to the development of predictive models of early clinical outcome.

The fundamental role of CT images analysis in the COVID-19 pandemic scenario also emerges in the manuscript by Biondi et al. [13]. The authors propose a study about the classification performance for COVID-19-patient prognosis from automatic Artificial Intelligence (AI) segmentation. They highlight the possibility of obtaining a reliable automated segmentation of lungs and of Ground-Glass Opacities areas in CT scans using non-supervised approaches and using this segmentation in a prediction pipeline for patient prognosis. Semi-supervised segmentation, implemented using a combination of non-supervised segmentation and feature extraction, seems to be a viable approach for patient stratification and could be leveraged to train more complex models. This would be useful in a high-demand situation similar to the current pandemic to support gold-standard segmentation for AI training.

The growing demand and use of AI, not only for quantitative imaging, but in several other areas of medicine, is highlighted by Avanzo et al. [14]. In particular, in their review, the authors summarize the main applications of AI in medical physics and express their point of view on the role and the involvement of Medical Physicists (MPs) in this evolving scenario by defining the challenges of AI in healthcare for the MPs and by describing the skills the MPs can offer in this field. 
Finally, the research toward the development or improvement of instruments and methods for accurate dose evaluation, both in external and internal dosimetry, confirmed to be very active, and various contributions of this Special Issue deal with this topic.

Arilli et al. [15] evaluate the combined use of the Integral Quality Monitor (IQM, iRT Systems GmbH, Koblenz, Germany) transmission detector and SoftDiso software (Best Medical Srl-Chianciano Terme, Italy) for in vivo dose monitoring for the simultaneous detection of delivery and patient setup errors in whole-breast irradiation. The authors show that the two devices provide complementary information and allow the fast detection of all types of errors. The proposed method represents a new strategy in Quality Assurance (QA) programs and would be an important step forward in the clinical routine to increase the quality of external breast irradiation.

The issue of in vivo dosimetry in charged particle radiation therapy is considered by Cirrone et al. [16]. The authors investigate the suitability of a new online, non-invasive, biasless detector for relative dose monitoring. The detector, protected by both a national Italian and an International patent, exploits the idea that when a beam current is injected into the body of a patient undergoing a charged particle therapy, the current itself can be collected using a conductive electrode in contact with the skin of patient. After characterizing the system in vitro, preliminary in vivo tests highlight the capability of the device in acting as beam monitoring during a proton-therapy treatment and its ability in the verification of the correct dose delivered among the various fractions of the treatment.

A radiation detector with a relatively longer history is the GEMPix, a detector developed at CERN a few years ago. It couples two technologies, the Gas Electron Multiplier as an amplifier for electric charges and four highly pixelated Timepix ASICs as readout. Leidner et al. [17] propose a comprehensive review about the medical applications of GEMPix. The authors describe examples of the use of this device for relative dose measurements in $\mathrm{X}$-ray radiation therapy and hadron therapy. Some preliminary measurements performed to check the capabilities of GEMPix in proton tomography are also illustrated, as well as the most recent application of the detector in the field of microdosimetry. A discussion about on-going and future developments concludes the review.

In the frame of internal dosimetry, the study by Milano et al. [18] aims at the validation of a platform, named MCID, based on patient-specific images and direct Monte Carlo (MC) simulations for internal dosimetry in the radioembolization of liver tumors with ${ }^{90}$ Y-labeled microspheres. The validated platform allows the fast implementation of a personalized MC dosimetry, based on patient imaging data. Additionally, the authors investigate the impact of tissue inhomogeneities on the dosimetric evaluation for the radioembolization treatment and the potential improvement of a MC approach in this therapy.

The same treatment strategy for liver lesions, based on percutaneous ablation using ${ }^{90} \mathrm{Y}$ microspheres embedded into a sealant matrix, is considered by D'Arienzo et al. [19]. In this manuscript, the absorbed dose per unit administered activity is assessed in small lesions by MC calculations considering a simplified geometry. Furthermore, an analytical formula derived from MC calculations that incorporates the absorbed fractions for ${ }^{90} \mathrm{Y}$ is proposed. The authors conclude that in a scenario of the intra-tumoral injection of microspheres, the proposed equation can be usefully employed in the treatment planning of spherical lesions of small size (down to $0.5 \mathrm{~cm}$ diameter) providing dose estimates in close agreement with MC calculations.

Lastly, under the main topic of dosimetry, the Special Issue includes the study by Gonzales et al. [20] which contribute to the development of a practical protocol for fingernail electron spin resonance (ESR) dosimetry useful for the routine monitoring of the extremity exposures of medical workers handling radiation sources. The authors investigate the effects of chemical treatment using dithiothreitol (DTT) on the changes of the ESR spectra in irradiated and unirradiated fingernails after a long storage time. The results show that the treatment of fingernail samples with DTT lead to a significant reduction of the background signal. They also point out that freezer storage of the samples is more effective than vacuum storage. Furthermore, after the DTT treatment, the fingernail samples provided 
good signal stability for both background and radiation-induced signals with less intraindividual variations.

\section{Conclusions}

In conclusion, we were very pleased to guest-edit this Special Issue that collects relevant contributions reflecting the increasingly widespread interest in medical physics and related applications.

We hope this Special Issue could reach the widest audience in the scientific community and contribute to boosting further scientific and technological advances into the intriguing world of medical physics and their multidisciplinary applications.

Finally, we wish this Special Issue may help the readers to conceive both new and improved ideas about the "Applications of Medical Physics" in their respective fields.

Author Contributions: Both Authors have contributed to the conceptualization, writing, review and editing of this manuscript. All authors have read and agreed to the published version of the manuscript.

Funding: This research received no external funding.

Acknowledgments: The Guest Editors would like to express their sincere thanks to all authors for their valuable contributions and to all peer-reviewers for their constructive comments and suggestions.

Conflicts of Interest: The authors declare no conflict of interest.

\section{References}

1. Crapanzano, R.; Secchi, V.; Villa, I. Co-Adjuvant Nanoparticles for Radiotherapy Treatments of Oncological Diseases. Appl. Sci. 2021, 11, 7073. [CrossRef]

2. Tai, H.-C.; Lee, J.; Huang, W.-C.; Liu, H.-C.; Chen, C.-H.; Huang, Y.-C.; Lee, C.-J.; Yun, C.-H.; Hsu, S.-M.; Chen, Y.-J. The Impact of Radiation to Epicardial Adipose Tissue on Prognosis of Esophageal Squamous Cell Carcinoma Receiving Neoadjuvant Chemoradiotherapy and Esophagectomy. Appl. Sci. 2021, 11, 4023. [CrossRef]

3. Jo, B.; Park, K.; Shin, D.; Lim, Y.K.; Jeong, J.H.; Lee, S.B.; Kim, H.-J.; Kim, H. Feasibility Study of Robust Optimization to Reduce Dose Delivery Uncertainty by Potential Applicator Displacements for a Cervix Brachytherapy. Appl. Sci. 2021, 11, 2592. [CrossRef]

4. Petringa, G.; Calvaruso, M.; Conte, V.; Bláha, P.; Bravatà, V.; Cammarata, F.P.; Cuttone, G.; Forte, G.I.; Keta, O.; Manti, L.; et al. Radiobiological Outcomes, Microdosimetric Evaluations and Monte Carlo Predictions in Eye Proton Therapy. Appl. Sci. 2021, 11, 8822. [CrossRef]

5. Urbano, N.; Scimeca, M.; Bonfiglio, R.; Mauriello, A.; Bonanno, E.; Schillaci, O. [99mTc]Tc-Sestamibi Bioaccumulation Can Induce Apoptosis in Breast Cancer Cells: Molecular and Clinical Perspectives. Appl. Sci. 2021, 11, 2733. [CrossRef]

6. Albano, D.; Loria, A.; Fanciullo, C.; Bruno, A.; Messina, C.; del Vecchio, A.; Sconfienza, L.M. Diagnostic Performance and Radiation Dose of the EOS System to Image Enchondromatosis: A Phantom Study. Appl. Sci. 2020, 10, 8941. [CrossRef]

7. Muhammad, N.A.; Kayun, Z.; Abu Hassan, H.; Wong, J.H.D.; Ng, K.H.; Karim, M.K.A. Evaluation of Organ Dose and Image Quality Metrics of Pediatric CT Chest-Abdomen-Pelvis (CAP) Examination: An Anthropomorphic Phantom Study. Appl. Sci. 2021, 11, 2047. [CrossRef]

8. Barca, P.; Marfisi, D.; Marzi, C.; Cozza, S.; Diciotti, S.; Traino, A.C.; Giannelli, M. A Voxel-Based Assessment of Noise Properties in Computed Tomography Imaging with the ASiR-V and ASiR Iterative Reconstruction Algorithms. Appl. Sci. 2021, 11, 6561. [CrossRef]

9. Savini, A.; Feliciani, G.; Amadori, M.; Rivetti, S.; Cremonesi, M.; Cesarini, F.; Licciardello, T.; Severi, D.; Ravaglia, V.; Vagheggini, A.; et al. The Role of Acquisition Angle in Digital Breast Tomosynthesis: A Texture Analysis Study. Appl. Sci. 2020, 10, 6047. [CrossRef]

10. Di Martino, F.; Barca, P.; Bortoli, E.; Giuliano, A.; Volterrani, D. Correction for the Partial Volume Effects (PVE) in Nuclear Medicine Imaging: A Post-Reconstruction Analytic Method. Appl. Sci. 2021, 11, 6460. [CrossRef]

11. Cusumano, D.; Catucci, F.; Romano, A.; Boldrini, L.; Piras, A.; Broggi, S.; Votta, C.; Placidi, L.; Nardini, M.; Chiloiro, G.; et al. Evaluation of an Early Regression Index (ERITCP) as Predictor of Pathological Complete Response in Cervical Cancer: A Pilot-Study. Appl. Sci. 2020, 10, 8001. [CrossRef]

12. Mazzilli, A.; Fiorino, C.; Loria, A.; Mori, M.; Esposito, P.G.; Palumbo, D.; de Cobelli, F.; del Vecchio, A. An Automatic Approach for Individual HU-Based Characterization of Lungs in COVID-19 Patients. Appl. Sci. 2021, 11, 1238. [CrossRef]

13. Biondi, R.; Curti, N.; Coppola, F.; Giampieri, E.; Vara, G.; Bartoletti, M.; Cattabriga, A.; Cocozza, M.A.; Ciccarese, F.; De Benedittis, C.; et al. Classification Performance for COVID Patient Prognosis from Automatic AI Segmentation-A Single-Center Study. Appl. Sci. 2021, 11, 5438. [CrossRef] 
14. Avanzo, M.; Trianni, A.; Botta, F.; Talamonti, C.; Stasi, M.; Iori, M. Artificial Intelligence and the Medical Physicist: Welcome to the Machine. Appl. Sci. 2021, 11, 1691. [CrossRef]

15. Arilli, C.; Wandael, Y.; Galeotti, C.; Marrazzo, L.; Calusi, S.; Grusio, M.; Desideri, I.; Fusi, F.; Piermattei, A.; Pallotta, S.; et al. Combined Use of a Transmission Detector and an EPID-Based In Vivo Dose Monitoring System in External Beam Whole Breast Irradiation: A Study with an Anthropomorphic Female Phantom. Appl. Sci. 2020, 10, 7611. [CrossRef]

16. Cirrone, G.A.P.; Amato, N.; Catalano, R.; Di Domenico, A.; Cuttone, G.; Lojacono, P.; Mazzaglia, A.; Pace, F.; Pittà, G.; Raffaele, L.; et al. On the Possibility to Use the Charge Imbalance in Patients Undergoing Radiotherapy: A New Online, In Vivo, Noninvasive Dose Monitoring System. Appl. Sci. 2021, 11, 7005. [CrossRef]

17. Leidner, J.; Murtas, F.; Silari, M. Medical Applications of the GEMPix. Appl. Sci. 2021, 11, 440. [CrossRef]

18. Milano, A.; Gil, A.V.; Fabrizi, E.; Cremonesi, M.; Veronese, I.; Gallo, S.; Lanconelli, N.; Faccini, R.; Pacilio, M. In Silico Validation of MCID Platform for Monte Carlo-Based Voxel Dosimetry Applied to 90Y-Radioembolization of Liver Malignancies. Appl. Sci. 2021, 11, 1939. [CrossRef]

19. D'Arienzo, M.; Sarnelli, A.; Mezzenga, E.; Chiacchiararelli, L.; Amato, A.; Romanelli, M.; Cianni, R.; Cremonesi, M.; Paganelli, G. Dosimetric Issues Associated with Percutaneous Ablation of Small Liver Lesions with 90Y. Appl. Sci. 2020, 10, 6605. [CrossRef]

20. Gonzales, C.A.B.; Taño, J.E.; Yasuda, H. An Attempt to Reduce the Background Free Radicals in Fingernails for Monitoring Accidental Hand Exposure of Medical Workers. Appl. Sci. 2020, 10, 8949. [CrossRef] 